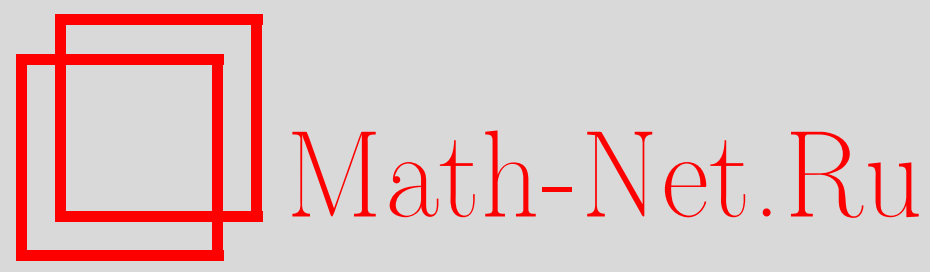

Л. А. Калякин, Асимптотический распад решения возмущенного уравнения Лиувилля, Матем. заметки, 2000, том 68, выпуск 2, 195-209

DOI: https://doi.org/10.4213/mzm938

Использование Общероссийского математического портала Math-Net.Ru подразумевает, что вы прочитали и согласны с пользовательским соглашением http://www . mathnet.ru/rus/agreement

Параметры загрузки:

IP : 18.208 .226 .222

26 апреля 2023 г., $17: 37: 08$

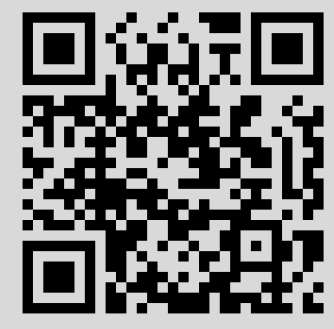




\section{АСИМПТОТИЧЕСКИЙ РАСПАД РЕШЕНИЯ ВОЗМУШЕННОГО УРАВНЕНИЯ ЛИУВИЛЛЯ}

\section{Л. А. Калякин}

Рассматривается задача Коши для возмущенного уравнения Лиувилля. Методом двухмасштабных разложений строится асимптотическое по параметру возмущения решение, пригодное до далеких времен. Основной результат - определение деформации главного члена асимптотики в масштабе медленного времени.

Библиография: 19 названий.

Введение. Асимптотическое интегрирование уравнений в частных производных, которые близки к уравнениям, интегрируемым точно, остается одной из привлекательных задач теории возмущений. Особый интерес представляют задачи о возмущении так назьваемых солитонных уравнений, поскольку последние тесно связаны с приложениями во многих областях физики и механики, [1]-[11]. В данной статье рассматривается возмущение нелинейного уравнения Лиувилля. Предлагается конструкция, которая обеспечивает асимптотическое интегрирование задачи Коши с любыми гладкими убывающими начальными данными.

Уравнение Лиувилля $\varphi_{t t}-\varphi_{x x}+8 \exp \varphi=0$ известно в физике как одна из моделей нелинейной теории поля [12], [13]. Популярность этого интегрируемого волнового уравнения во многом обязана наличию формулы для общего решения

$$
\varphi(x, t)=\ln \frac{r_{+}^{\prime}\left(s^{+}\right) r_{-}^{\prime}\left(s^{-}\right)}{r^{2}\left(s^{+}, s^{-}\right)}, \quad r=r_{+}\left(s^{+}\right)+r_{-}\left(s^{-}\right), \quad s^{ \pm}=x \pm t .
$$

Штрихи всюду означают дифференцирование по пространственной переменной $x$ либо по конусной переменной $s^{ \pm}$. Эта формула, полученная еще Лиувиллем [14], содержит два функциональных произвола $r_{ \pm}$, которые обеспечивают решение задачи Коши.

Объектом данного исследования является задача Коши для уравнения с малым возмущением

$$
\partial_{t}^{2} u-\partial_{x}^{2} u+8 \exp u=\varepsilon \boldsymbol{F}[u], \quad x \in \mathbb{R}, \quad t>0, \quad 0<\varepsilon \ll 1 .
$$

Предполагается, что начальные данные

$$
\left.u\right|_{t=0}=\psi_{0}(x),\left.\quad \partial_{t} u\right|_{t=0}=\psi_{1}(x), \quad x \in \mathbb{R},
$$

Работа выполнена при поддержке Российского фонда фундаментальных исследований, грант № 97-01-00459. 
быстро стремятся к нулю на бесконечности: $\psi_{0}, \psi_{1}(x)=O\left(x^{-N}\right),|x| \rightarrow \infty$ для любого $N$. Здесь и всюду в дальнейшем все функции и асимптотики являются гладкими, т. е. бесконечно дифференцируемыми. Оператор возмушения задается парой гладких функций двух переменных в виде

$$
\boldsymbol{F}[u]=\partial_{x} F_{1}\left(\partial_{x} u, \partial_{t} u\right)+\partial_{t} F_{2}\left(\partial_{x} u, \partial_{t} u\right)
$$

Ставится задача об асимптотическом приближении решения $u(x, t ; \varepsilon)$ при $\varepsilon \rightarrow 0$ равномерно в широкой полосе $x \in \mathbb{R}, 0<t \leqslant O\left(\varepsilon^{-1}\right)$.

Решение невозмущенной задачи в своей асимптотике на бесконечности распадается на две простые волны, расходящиеся на фоне линейно убьвающего главного члена: $\varphi(x, t)=-4 t+A_{ \pm}\left(s^{ \pm}\right)+O\left(\left(s^{\mp}\right)^{-N}\right), s^{\mp} \rightarrow \mp \infty$. При возмущении этот распад сохраняется в главном члене асимптотики по $\varepsilon \rightarrow 0$. Влияние возмущения проявляется в слабой деформации волн $A_{ \pm}=A_{ \pm}\left(s^{ \pm}, \tau\right)$ в масштабе $\tau=\varepsilon t$ и приводит к эффектам порядка $O(1)$ на далеких временах $t \approx \varepsilon^{-1}$. Такая деформация описьвается уравнениями первого порядка в частных производных

$$
\pm 2 \partial_{\tau} A_{ \pm}=H_{ \pm}\left(\partial_{s} A_{ \pm}\right)
$$

где $H_{ \pm}(B)=F_{1}(B,-4 \pm B)-F_{1}(0,-4) \pm F_{2}(B,-4 \pm B) \mp F_{2}(0,-4)$. Начальные данные для $A_{ \pm}$вычисляются из невозмущенного решения. Поэтому эффективность предлагаемого подхода в основном определяется эффективностью решения задачи Коши для невозмущенного уравнения.

1. Вид формального решения задачи Коши. Простейшая конструкция в виде прямого ряда теории возмущений

$$
u \approx \sum_{n=0}^{\infty} \varepsilon^{n}{ }_{u}^{n}(x, t), \quad \varepsilon \rightarrow 0
$$

дает асимптотику решения равномерно в любом замкнутом характеристическом треугольнике, который не зависит от $\varepsilon$. В качестве главного члена берется решение невозмущенного уравнения $\stackrel{0}{u}=\varphi(x, t)$. Возмущение учитьвается в поправках $u_{n}(n \geqslant 1)$, которые определяются из линеаризованных уравнений. Сингулярньй характер возмущения и непригодность прямого разложения связаны с большим промежутком времени. Как обычно в подобных задачах, в поправках $\stackrel{n}{u}$ обнаруживаются секулярные члены $O\left(t^{n}\right)$, которые делают непригодньм разложение $(*)$ на далеких временах $t \approx \varepsilon^{-1}$.

На далеких временах малые возмущения проявляются в главном члене асимптотики, что обнаруживается в параметрах $r_{ \pm}$в виде зависимости от медленного времени $\tau=\varepsilon t$. Определение функций $r_{ \pm}=r_{ \pm}\left(s^{ \pm}, \tau\right)$ при $\tau=O(1)$, исходя из требования отсутствия секулярных членов в поправках, является основной задачей конструирования формального асимптотического решения ( $\Phi \mathrm{AP})$.

ОПРЕДЕЛЕНИЕ 1 . Под $\Phi A P$ порядка $N$ понимается функция $U_{N}(x, t ; \varepsilon)$, которая удовлетворяет уравнениям $(0.2),(0.3)$ с точностью $o\left(\varepsilon^{N}\right), \varepsilon \rightarrow 0$, равномерно в широкой полосе $x \in \mathbb{R}, 0<t \leqslant O\left(\varepsilon^{-1}\right)$. 
В данной работе мы ограничиваемся построением $\Phi$ АР второго порядка. Предлагаемьй нами подход напоминает известньй метод Крылова-Боголюбова [15]. В качестве $\Phi А Р$ берется конечный отрезок ряда

$$
u(x, t ; \varepsilon) \approx \sum_{n=0}^{\infty} \varepsilon^{n} u(x, t, \tau ; \varepsilon),
$$

в коэффициентах которого допускается специфическая зависимость от медленного времени $\tau=\varepsilon t$ и параметра $\varepsilon$. Для главного члена используется решение невозмущенного уравнения

$$
\stackrel{0}{u}=\ln \left(\frac{r_{+}^{\prime} r_{-}^{\prime}}{r^{2}}\right) \quad\left(r=r_{+}+r_{-}\right),
$$

которое параметризуется парой функций $r_{ \pm}\left(s^{ \pm}, \tau ; \varepsilon\right)$, зависящих дополнительно от $\tau=\varepsilon t$ и от малого параметра $\varepsilon$. Для них строится своя асимптотика

$$
r_{ \pm}\left(s^{ \pm}, \tau ; \varepsilon\right) \approx \sum_{n=0}^{\infty} \varepsilon^{n} r_{ \pm}^{n}\left(s^{ \pm}, \tau\right) .
$$

Конструирование $\Phi$ АР сводится к нахождению коэффициентов разложения $\stackrel{n}{u}, \stackrel{n}{r} \pm$. Поправки $\stackrel{n}{u}$ берутся из рекуррентной системы линеаризованных уравнений

$$
\partial_{t}^{2} \stackrel{n}{u}-\partial_{x}^{2} \stackrel{n}{u}+8 \stackrel{n}{u} \exp (\stackrel{0}{u})=\stackrel{n}{f}(x, t ; \varepsilon), \quad x \in \mathbb{R}, \quad t>0
$$

Правые части определяются через предыдущие приближения; например,

$$
\stackrel{1}{f}=\boldsymbol{F}[\stackrel{0}{u}]-2 \partial_{\tau} \partial_{t} \stackrel{0}{u}, \quad \stackrel{2}{f}=\delta \boldsymbol{F}[\stackrel{0}{u}] \stackrel{1}{u}+\delta_{u_{t}} \boldsymbol{F}[\stackrel{0}{u}] \partial_{\tau} \stackrel{0}{u}-4(\stackrel{1}{u})^{2} \exp \stackrel{0}{u}-2 \partial_{\tau} \partial_{t} \stackrel{1}{u}-\partial_{\tau}^{2} \stackrel{0}{u} .
$$

Эти уравнения обеспечивают асимптотическое вьполнение исходного уравнения (0.2). Начальные условия (0.3) удовлетворяются за счет подходящего выбора функций $r_{ \pm}$.

Эффективность всей конструкции основана на явных формулах для решений линеаризованного уравнения. Эти решения вьписьваются через интегралы, и дело сводится к конструированию коэффициентов $\stackrel{n}{r} \pm$.

Так же, как в классическом методе Крылова-Боголюбова, уравнения, описывающие медленную деформацию параметров $r_{ \pm}$, можно получить из требований ограниченности поправок $\stackrel{n}{u}$ в быстрых переменных $x, t$. Получаемые таким способом уравнения для $\stackrel{n}{r} \pm$ выглядят довольно сложно. Поэтому мы предлагаем использовать для главного члена другую параметризацию $\stackrel{0}{u}=\Phi\left[A_{+}, A_{-}\right]-$через пару функций $A_{ \pm}\left(s^{ \pm}\right)$, которые представляют собой асимптотику на бесконечности решения уравнения Лиувилля. На этом пути получаются хорошо знакомые уравнения первого порядка $(0.5)$ с ясной геометрической интерпретацией. Они описывают медленную деформацию (под влиянием возмущения) простых волн, на которые распадается в бесконечности решение невозмущенного уравнения Лиувилля. Связь пары $A_{ \pm} \mathrm{c} r_{ \pm}$задается явными формулами, которые представляют собой основной результат следующего пункта. 
2. Асимптотика решения нелинейного уравнения Лиувилля. Решение задачи Коши для невозмущенного уравнения с использованием формулы (0.1) сводится $\mathrm{K}$ определению пары функций $r_{ \pm}(x)$ [13]. Начальные условия представляют собой систему дифференциальных уравнений третьего порядка и могут быть преобразованы к паре уравнений, которые заменой $r_{ \pm}^{\prime}=w / \rho_{ \pm}^{2}$ (для произвольного $w=$ const $\neq 0$ ) линеаризуются:

$$
\rho_{ \pm}^{\prime \prime}-\Psi_{ \pm}(x) \rho_{ \pm}=0
$$

Потенциалы здесь связаны с начальными данными:

$$
\Psi_{ \pm}(x)=\left[\exp \left(\psi_{0}\right)+\left(\frac{\psi_{0}^{\prime} \pm \psi_{1}}{4}\right)^{2}-\frac{1}{4}\left(\psi_{0}^{\prime} \pm \psi_{1}\right)^{\prime}\right]
$$

Требуемые в (0.1) функции выписьваются в виде $r_{ \pm}(x)=\widetilde{\rho}_{ \pm}(x) / \rho_{ \pm}(x)$ через линейно независимые решения с вронскианом $W\left(\rho_{ \pm}, \widetilde{\rho}_{ \pm}\right)=w$. При этом одна пара функций, например $\rho_{+}, \widetilde{\rho}_{+}$, может быть выбрана из уравнений (2.1) произвольно с четырьмя константами интегрирования. Вторая пара определяется конечными формулами, в которые превращаются начальные уравнения

$$
\begin{aligned}
& \rho_{-}=-\exp \left(-\frac{\psi_{0}}{2}\right)\left[\rho_{+}^{\prime}+\left(\psi_{0}^{\prime}+\psi_{1}\right) \frac{\rho_{+}}{4}\right] \\
& \widetilde{\rho}_{-}=\exp \left(-\frac{\psi_{0}}{2}\right)\left[\widetilde{\rho}_{+}^{\prime}+\left(\psi_{0}^{\prime}+\psi_{1}\right) \frac{\widetilde{\rho}_{+}}{4}\right] .
\end{aligned}
$$

Константы интегрирования в $\rho_{+}, \widetilde{\rho}_{+}$соответствуют трехпараметрическому произволу в преобразовании Бианки [16]

$$
\widetilde{r}_{ \pm}=\frac{ \pm a r_{ \pm}+b}{c r_{ \pm} \pm d} \quad(b c-a d=w)
$$

и неопределенности общего множителя $w$, с точностью до которых определяются функции $r_{ \pm}$; от этих констант не зависит решение, определяемое по формуле $(0.1)$.

ЛЕмма 2.1. Пусть гладкие начальные функиии быстро стремятся $к$ нулю на бесконечности: для всех $N \quad \psi_{0}(x), \psi_{1}(x)=O\left(x^{-N}\right),|x| \rightarrow \infty$, и уравнения (2.1) не находятся на спектре. Тогда решение уравнения Лиувилля с такими начальными данными имеет асимптотику в характерных направлениях на бесконечности в виде пары расходящихся волн на фоне линейно убывающего главного члена:

$$
\varphi(x, t)=-4 t+A_{ \pm}\left(s^{ \pm}\right)+O\left(\left(s^{\mp}\right)^{-N}\right)+O\left(e^{-4 t}\right), \quad s^{\mp} \rightarrow \mp \infty, \quad t \rightarrow \infty .
$$

Эти волны имеют согласованную асимптотику на бесконечности:

$$
A_{ \pm}(s)= \begin{cases}-\ln a^{2}+O\left(s^{-N}\right), & s \rightarrow \pm \infty(a=\text { const } \neq 0) \\ O\left(s^{-N}\right), & s \rightarrow \mp \infty .\end{cases}
$$


ПояснЕниЕ. Особенностью уравнения Лиувилля является наличие ненулевого решения с нулевьми начальными данными. Неубьвающий член асимптотики такого решения, равный $-4 t$, образует тот фон, по которому разбегаются волны $A_{ \pm}(x \pm t)$, обязанные ненулевым начальным данным.

ДокАЗАТЕЛЬСтво лЕммы. Зафиксируем два решения $\rho_{+}, \widetilde{\rho}_{+}$уравнений $(2.1)$ условиями убывания на $\pm \infty$ соответственно. Поскольку потенциалы $(2.2)$ быстро стабилизируются на бесконечности к единице, то имеются экспоненциально убьваюшие решения. С учетом формул (2.3), (2.4) асимптотика вьписывается сразу для обеих пар

$$
\begin{array}{ll}
\rho_{ \pm}=\exp (-x)\left[1+O\left(x^{-N}\right)\right], & x \rightarrow+\infty \\
\tilde{\rho}_{ \pm}=\exp (x)\left[1+O\left(x^{-N}\right)\right], & x \rightarrow-\infty
\end{array}
$$

Каждая из этих функций на противоположной бесконечности экспоненциально растет, если уравнения (2.1) не на спектре:

$$
\begin{array}{ll}
\rho_{ \pm}=a \exp (-x)\left[1+O\left(x^{-N}\right)\right], & x \rightarrow-\infty, \\
\tilde{\rho}_{ \pm}=a \exp (x)\left[1+O\left(x^{-N}\right)\right], & x \rightarrow+\infty .
\end{array}
$$

Константа $a$ одна и та же в силу постоянства вронскиана и формул связи $(2.3),(2.4)$; она определяется начальньми данными и условием $W\left(\rho_{ \pm}, \widetilde{\rho}_{ \pm}\right)=2 a$. После этого немедленно выписьваются асимптотики

$$
\begin{array}{ll}
r_{ \pm}(x)=\left(\frac{1}{a}\right) \exp (2 x)\left[1+O\left(x^{-N}\right)\right], & x \rightarrow-\infty \\
r_{ \pm}(x)=a \exp (2 x)\left[1+O\left(x^{-N}\right)\right], & x \rightarrow+\infty .
\end{array}
$$

Рассматриваемое нами решение (0.1) представим в виде

$$
\varphi(x, t)=\ln e^{-4 t} \frac{\exp \left(-2 s^{+}\right) r_{+}^{\prime}\left(s^{+}\right) \exp \left(-2 s^{-}\right) r_{-}^{\prime}\left(s^{-}\right)}{\left[\exp \left(-2 s^{+}\right) r_{+}\left(s^{+}\right)+\exp \left(-2 s^{-}\right) r_{-}\left(s^{-}\right) e^{-4 t}\right]^{2}} .
$$

Отсюда получается требуемая асимптотика при $s^{\mp} \rightarrow \mp \infty$ вдоль характерных направлений $s^{ \pm}=$const $\mathrm{c}$ функциями

$$
\begin{aligned}
& A_{+}\left(s^{+}\right)=\ln \left[\frac{\exp \left(2 s^{+}\right) r_{+}^{\prime}\left(s^{+}\right)}{a r_{+}^{2}\left(s^{+}\right)}\right] \\
& A_{-}\left(s^{-}\right)=\ln \left[\frac{\exp \left(-2 s^{-}\right) r_{-}^{\prime}\left(s^{-}\right)}{a}\right] .
\end{aligned}
$$

Формулы $(2.9),(2.10)$ имеют место лишь для конкретного выбора пары $r_{ \pm}\left(s^{ \pm}\right)$(с условиями $(2.7),(2.8))$. Для другой пары, получаемой по преобразованию Бианки, эти соотношения будут другими. Однако, функции $A_{ \pm}\left(s^{ \pm}\right)$от выбора пары $r_{ \pm}$не зависят в силу единственности решения задачи Коши [17] и его асимптотического разложения. 
СлЕДСтвиЕ 2.1. Решение уравнения Лиувилля, стремящееся $\kappa 0$ при $x \rightarrow \pm \infty$, однозначно определяется парой гладких функиий $A_{ \pm}\left(s^{ \pm}\right)$, которые обладают свойством (2.6).

ДокАЗАТЕЛЬСТво. Соотношения (2.9), (2.10) представляют собой дифференциальные уравнения для функций $1 / r_{+}(x), r_{-}(x)$. Будучи дополнены убывающими краевьми условиями из (2.8) и (2.7) соответственно, они однозначно определяют эти функции, а следовательно, и решение уравнения Лиувилля в форме (0.1). В полном объеме свойства $(2.8),(2.7)$ для $r_{ \pm}$вьполняются в силу согласования асимптоти $(2.6)$.

Неопределенности в функциях $r_{ \pm}$, связанные с преобразованием Бианки, здесь не видны, поскольку уравнения $(2.9),(2.10)$ вьведены для конкретных $r_{ \pm}(x)$ с вполне определенной асимптотикой на бесконечности. При других краевых условиях на $r_{ \pm}$соотношения $(2.9),(2.10)$ имеют другой вид и получаются другие функции $r_{ \pm}(x)$. Однако, pешение $\varphi(x, t)$ зависит только от пары $A_{ \pm}(x)$. В самом деле, для любой $\varphi(x, t)$ представление $(0.1)$ через пару $r_{ \pm}$с условиями $(2.7),(2.8)$ однозначно определяется функциями $A_{ \pm}(x)$ из уравнений $(2.9),(2.10)$, как показано вьше.

Таким образом, решение уравнение Лиувилля параметризуется парой функций $A_{ \pm}\left(s^{ \pm}\right)$. Такую параметризацию можно записать в операторной форме

$$
\varphi=\Phi\left[A_{+}, A_{-}\right] \equiv \ln \frac{r_{+}^{\prime} r_{-}^{\prime}}{\left(r_{+}+r_{-}\right)^{2}}
$$

имея в виду, что $r_{ \pm}$определяются через $A_{ \pm}$из уравнений $(2.7)-(2.10)$. Отметим, что условие (2.6) выделяет класс функций $A_{ \pm}$с согласованной асимптотикой, на котором определен оператор $\Phi\left[A_{+}, A_{-}\right]$.

3. Решение линеаризованного уравнения Лиувилля. Рассматривается задача Коши для линеаризованного уравнения

$$
\begin{gathered}
\partial_{t}^{2} u-\partial_{x}^{2} u+8 u \exp (\varphi)=f\left(s^{+}, s^{-}\right), \quad s^{ \pm}=x \pm t \\
\left.u\right|_{t=0}=\psi_{0}(x),\left.\quad \partial_{t} u\right|_{t=0}=\psi_{1}(x)
\end{gathered}
$$

Решение выписьвается через интегралы, и его можно проверить непосредственной подстановкой. Тем не менее, мы вкратце опишем путь, ведуший к этим ответам. Основой является формула для общего решения однородного уравнения

$$
u(x, t)=\frac{j_{+}^{\prime}\left(s^{+}\right)}{r_{+}^{\prime}\left(s^{+}\right)}+\frac{j_{-}^{\prime}\left(s^{-}\right)}{r_{-}^{\prime}\left(s^{-}\right)}-2 \frac{j_{+}\left(s^{+}\right)+j_{-}\left(s^{-}\right)}{r\left(s^{+}, s^{-}\right)}, \quad r=r_{+}\left(s^{+}\right)+r_{-}\left(s^{-}\right) .
$$

Эта формула получается вариацией общего решения уравнения Лиувилля (0.1) и содержит пару функциональных произволов $j_{ \pm}\left(s^{ \pm}\right)$. Начальные условия (3.2) дают систему уравнений третьего порядка для функций $j_{ \pm}(x)$. Эти уравнения легко интегрируются, в результате получается интегральное представление решения, похожее на формулу Даламбера

$$
u(x, t)=\frac{1}{2}\left[\psi_{0}\left(s^{-}\right)+\psi_{0}\left(s^{+}\right)\right]+\int_{s^{-}}^{s^{+}}\left[K_{0}\left(s^{+}, s^{-}, \sigma\right) \psi_{0}(\sigma)+K\left(s^{+}, s^{-}, \sigma, \sigma\right) \psi_{1}(\sigma)\right] d \sigma
$$


$\left(s^{ \pm}=x \pm t\right)$. Ядра выражаются через известные функции $r_{ \pm}$. Одно из них имеет вид

$$
\begin{aligned}
K_{0}\left(s^{+}, s^{-}, \sigma\right)= & \frac{1}{r\left(s_{+}, s_{-}\right) r^{2}(\sigma, \sigma)}\left\{r_{+}\left(s^{+}\right) r_{-}\left(s^{-}\right)\left[r_{+}(\sigma)-r_{-}(\sigma)\right]^{\prime}\right. \\
& \left.-\left[r_{+}\left(s^{+}\right)-r_{-}\left(s^{-}\right)\right]\left[r_{+}(\sigma) r_{-}(\sigma)\right]^{\prime}+r_{+}^{2}(\sigma) r_{-}^{\prime}(\sigma)-r_{-}^{2}(\sigma) r_{+}^{\prime}(\sigma)\right\} .
\end{aligned}
$$

Другое будет выписано ниже при решении неоднородного уравнения.

Лемма 3.1. Решение неоднородного линеаризованного уравнения (3.1) с гладкой правой частью и с однородными начальными данными выписывается через интеграл по характеристическому конусу, который в конусных переменных имеет вид

$$
u(x, t)=\frac{1}{2} \int_{s^{-}}^{s^{+}} \int_{s^{-}}^{\sigma^{+}} K\left(s^{+}, s^{-}, \sigma^{+}, \sigma^{-}\right) f\left(\sigma^{+}, \sigma^{-}\right) d \sigma^{-} d \sigma^{+}, \quad s^{ \pm}=x \pm t .
$$

Ядро выражается через функции $r_{ \pm}$, параметризующие решение исходного нелинейного уравнения,

$$
\begin{aligned}
K\left(s^{+}, s^{-}, \sigma^{+}, \sigma^{-}\right)= & \frac{1}{r\left(s^{+}, s^{-}\right) r\left(\sigma^{+}, \sigma^{-}\right)}\left\{r_{+}\left(s^{+}\right) r_{-}\left(s^{-}\right)+r_{+}\left(\sigma^{+}\right) r_{-}\left(\sigma^{-}\right)\right. \\
& \left.+\frac{1}{2}\left[r_{+}\left(s^{+}\right)-r_{-}\left(s^{-}\right)\right]\left[r_{+}\left(\sigma^{+}\right)-r_{-}\left(\sigma^{-}\right)\right]\right\} .
\end{aligned}
$$

Рассуждения, которые приводят к формуле (3.4), аналогичны методу вариации произвольных постоянных. Решение неоднородного уравнения строится в форме, похожей на (3.3),

$$
u(x, t)=\frac{j_{+}^{\prime}\left(s^{+}, t\right)}{r_{+}^{\prime}\left(s^{+}\right)}+\frac{j_{-}^{\prime}\left(s^{-}, t\right)}{r_{-}^{\prime}\left(s^{-}\right)}-2 \frac{j_{+}\left(s^{+}, t\right)+j_{-}\left(s^{-}, t\right)}{r\left(s^{+}, s^{-}\right)} \equiv U\left(s^{+}, s^{-}, t\right),
$$

с парой функций $j_{ \pm}\left(s^{ \pm}, t\right)$, зависящих от двух переменных. Определение этих функций из уравнения (3.1) при дополнительном требовании $\partial_{t} U\left(s^{+}, s^{-}, t\right)=0$ приводит к паре обыкновенных дифференциальных (по $x)$ уравнений для $J(x, t)=\partial_{t} j_{ \pm}\left(s^{ \pm}, t\right)$, $s^{ \pm}=x \pm t$. Эти уравнения совпадают с теми, что были в начальной задаче в частном случае $\psi_{0}=0, \psi_{1}=f$. После их интегрирования и восстановления функций $j_{ \pm}\left(s^{ \pm}, t\right)$ из производных получается формула (3.4) в виде интеграла по характеристическому конусу.

Из полученных формул можно извлечь асимптотику решения $u(x, t)$ на бесконечности, исходя из заданной асимптотики правых частей. Проще всего строится частное быстроубывающее решение.

ЛЕмМА 3.2. Если правая часть уравнения (3.1) быстро убывает, т.е.

$$
f\left(s^{+}, s^{-}\right)=O\left(\left(\left|s^{+}\right|+\left|s^{-}\right|\right)^{-N}\right), \quad\left|s^{+}\right|+\left|s^{-}\right| \rightarrow \infty, \quad \forall N,
$$

то существует решение, которое также быстро убывает в верхней полуплоскости $t>0$ :

$$
u(x, t)=O\left(\left(\left|s^{+}\right|+\left|s^{-}\right|\right)^{-N}\right), \quad\left|s^{+}\right|+\left|s^{-}\right| \rightarrow \infty, \quad \forall N .
$$


ДокАЗАТЕльство. Берем частное решение, представимое через интеграл по световому конусу с вершиной в точке $s^{+}, s^{-}$:

$$
u(x, t)=-\frac{1}{2} \int_{\infty}^{s^{+}} \int_{-\infty}^{s^{-}} K\left(s^{+}, s^{-}, \sigma^{+}, \sigma^{-}\right) f\left(\sigma^{+}, \sigma^{-}\right) d \sigma^{-} d \sigma^{+} .
$$

Легко проверить, что если конус расположен в верхней полуплоскости $s^{+}>s^{-}$, то в рассматриваемой области интегрирования $\left\{\sigma^{+}>s^{+}, \sigma^{-}<s^{-}\right\}$ядро $K\left(s^{+}, s^{-}, \sigma^{+}, \sigma^{-}\right)$ ограничено равномерно по всем переменным. Ввиду быстрого убывания подынтегральной функции интеграл (3.6) оценивается через $O\left(R^{-N}\right)$, где $R$ - расстояние от начала координат до конуса. Для конуса в верхней полуплоскости величина $R$ имеет порядок расстояния до вершины $R=O\left(\left|s^{+}\right|+\left|s^{-}\right|\right)$. Тем самым лемма доказана.

ЛЕмМА 3.3. Если правая часть быстро убывает, т.е.

$$
f\left(s^{+}, s^{-}\right)=O\left(\left(\left|s^{+}\right|+\left|s^{-}\right|\right)^{-N}\right), \quad\left|s^{+}\right|+\left|s^{-}\right| \rightarrow \infty, \quad \forall N
$$

то решение уравнения (3.1) с однородными начальными условиями в своей асимптотике на бесконечности в характерных направлениях быстро выходит на пару простых волн:

$$
u(x, t)=U_{ \pm}\left(s^{ \pm}\right)+O\left(\left(s^{\mp}\right)^{-N}\right), \quad s^{\mp} \rightarrow \mp \infty .
$$

Эта асимптотика является равномерной в секторах $\left|s^{ \pm}\right|<\mp \gamma s^{\mp}$ (при всех $\gamma=$ const $>0$ ), которые перекрываются при $\gamma>1$. Функиии $U_{ \pm}\left(s^{ \pm}\right)$имеют согласованную асимптотику на бесконечности

$$
U_{ \pm}(s)= \begin{cases}U_{\infty}+O\left(s^{-N}\right), & s \rightarrow \pm \infty, U_{\infty}=\text { const } \\ O\left(s^{-N}\right), & s \rightarrow \mp \infty\end{cases}
$$

ДоКАЗАТЕЛЬСТво вытекает из явной формулы (3.4), в которую подставляется ядро (3.5) и учитьваются асимптотики (2.6), (2.7). При этом область интегрирования в виде характеристического конуса в асимптотическом пределе переходит в сектор. В результате получается требуемая асимптотика (3.7) с функциями

$$
U_{ \pm}\left(s^{ \pm}\right)=\frac{1}{2} \int_{\mp \infty}^{s^{ \pm}} d \sigma^{ \pm} \int_{\mp \infty}^{\sigma^{ \pm}} d \sigma^{\mp} K_{ \pm}\left(s^{ \pm}, \sigma^{+}, \sigma^{-}\right) f\left(\sigma^{+}, \sigma^{-}\right)
$$

где ядра определяются формулами:

$$
\begin{aligned}
& K_{+}=\frac{1}{r\left(\sigma^{+}, \sigma^{-}\right)}\left\{\frac{1}{2}\left[r_{+}\left(\sigma^{+}\right)-r_{-}\left(\sigma^{-}\right)\right]+\frac{r_{+}\left(\sigma^{+}\right) r_{-}\left(\sigma^{-}\right)}{r_{+}\left(s^{+}\right)}\right\} \\
& K_{-}=\frac{1}{r\left(\sigma^{+}, \sigma^{-}\right)}\left\{\frac{1}{2}\left[r_{+}\left(\sigma^{+}\right)-r_{-}\left(\sigma^{-}\right)\right]+r_{-}\left(s^{-}\right)\right\}
\end{aligned}
$$

Константа $U_{\infty}$ выражается через интеграл по полуплоскости типа (3.4). 
ЗАмЕЧАниЕ 1. Формулы для ядер $K, K_{0}$ инвариантны относительно преобразования Бианки.

ЗАмЕЧАнИЕ 2. Функции $U_{ \pm}$определяются однозначно в силу единственности асимптотики и не зависят от выбора конкретной пары $r_{ \pm}$.

ЗАмЕчАниЕ 3. Однако, представления (3.8)-(3.10) для функций $U_{ \pm}$получены для $r_{ \pm}$с определенной асимптотикой $(2.7),(2.8)$. Эти представления не инвариантны; для другой пары $r_{ \pm}$формулы $(3.9),(3.10)$ имеют другой вид в соответствии с преобразованием Бианки.

4. Построение главного члена $\mathbf{\Phi А P . ~ И с х о д н а я ~ и д е я ~ с о с т о и т ~ в ~ п р и в л е ч е н и и ~ п а - ~}$ ры функций $A_{ \pm}$из асимптотики на бесконечности для параметризации решения уравнения Лиувилля. Представление главного члена $\Phi$ АР через такое решение $\stackrel{0}{u}=\Phi\left[A_{+}, A_{-}\right]$ основано на формуле $(2.11)$, в которой определяется оператор $\Phi$.

Вторая идея происходит из метода двух масштабов: в параметрах $A_{ \pm}$допускается зависимость от медленного времени: $A_{ \pm}=A_{ \pm}\left(s^{ \pm}, \tau\right), \tau=\varepsilon t$. Для этой пары функций задаются начальные значения

$$
A_{ \pm}(s, 0)=A_{0, \pm}(s)
$$

из требований вьполнения исходных начальных условий (0.3) в главном члене $\Phi$ АР. Функции $A_{0, \pm}(x)$ вычисляются через исходные данные $\psi_{0}(x), \psi_{1}(x)$ в соответствии c (2.9), (2.10).

Записанные в конусных переменных начальные функции $A_{0, \pm}(x \pm t)$ вьглядят как простые волны и представляют собой существенную часть неубывающей при $t \rightarrow \infty$ асимптотики для невозмущенного решения Лиувилля. Для возмущенного решения их можно рассматривать как ростки главного члена $\Phi$ АР на выходе из внутреннего слоя (при $t \gg 1)$. Эффекты возмущения проявляются на этих волнах на далеких временах и могут быть обнаружены в виде медленной деформации их в масштабе $\tau=\varepsilon t$. Построение главного члена $\Phi$ АР сводится к получению уравнений, которые описьвают эволюцию по $\tau$ функций $A_{ \pm}\left(s^{ \pm}, \tau\right)$.

Уравнения для них получаются (как обычно в методе двухмасштабных разложений) из секулярного условия: поправка в $\Phi$ АР мала: $\varepsilon u=o(1), \varepsilon \rightarrow 0$, равномерно вплоть до далеких времен $t=O\left(\varepsilon^{-1}\right)$. Напомним, что поправки определяются из линеаризованных уравнений (1.4), в которых медленная переменная $\tau$ входит в качестве параметра. В уравнении для первой поправки правая часть выражается по формуле

$$
\stackrel{1}{f}(x, t, \tau)=\boldsymbol{F}[\stackrel{0}{u}]-2 \partial_{\tau} \partial_{t} \stackrel{0}{u}
$$

и ввиду неубывающей асимптотики главного члена $\stackrel{0}{u}$, вообще говоря, не убьвает на бесконечности в характерных направлениях при $s^{ \pm} \rightarrow \pm \infty$. Поскольку решение линеаризованного уравнения выражается через интеграл по конусу от правой части, то из формулы (3.4) нетрудно усмотреть, что функция $\stackrel{1}{u}(x, t, \tau)$ может линейно расти в тех же направлениях при $s^{ \pm} \rightarrow \pm \infty$. Из-за этого на далеких временах $t \approx \varepsilon^{-1}$ поправка $\varepsilon \dot{1}$ оказьвается порядка единицы, что сравнимо с существенной частью главного члена $\Phi А Р$. Исключение таких секулярностей возможно за счет неопределенностей в параметрах $A_{ \pm}$, остающихся пока при $\tau>0$. Для этого в правой части $f$ следует выделить 
и приравнять к нулю неубьвающие члены асимптотики. Таким образом получаются уравнения, в общем случае нелинейные, структура которых определяется возмущением

$$
\pm 2 \partial_{\tau} \partial_{s} A_{ \pm}=\boldsymbol{F}\left[-4 t+A_{ \pm}\right]
$$

В постановке задачи для функций $A_{ \pm}$используются условия на бесконечности, которые получаются из соображений согласования с асимптотикой невозмущенного решения (2.6):

$$
A_{ \pm}\left(s^{ \pm}, \tau\right) \rightarrow 0 \quad \text { при } \quad s^{ \pm} \rightarrow \mp \infty .
$$

В случае возмущений вида (0.4) соотношения (4.2) можно проинтегрировать по $s$. С учетом условий (4.3) получаются уравнения первого порядка

$$
\pm 2 \partial_{\tau} A_{ \pm}=H_{ \pm}\left(\partial_{s} A_{ \pm}\right)
$$

с функциями $H_{ \pm}(B)=F_{1}(B,-4 \pm B)-F_{1}(0,-4) \pm F_{2}(B,-4 \pm B) \mp F_{2}(0,-4)$.

Лемма 4.1. 1. Если оператор возмущения имеет вид (0.4), то уравнения медленных деформаций (4.2) с условиями (4.1), (4.3) имеют единственное решение в классе гладких функиий на некотором промежутке (локально в медленном времени) $\tau \in\left[0, \tau_{0}\right] .2$. При этом главный член ФАР определяется через решение Лиувилля (2.11), зависящее от параметра $\tau \in\left[0, \tau_{0}\right]$. 3. Первая поправка $\stackrel{1}{u}$, определяемая из уравнения (1.4), будет ограничена равномерно по $x, t$.

ДокАзАтЕЛьство. Локальная разрешимость задачи Коши (4.1), (4.4) не вызывает сомнения. Это обеспечивает однозначное определение функций $A_{ \pm}\left(s^{ \pm}, \tau\right)$ на некотором конечном промежутке по $\tau$.

Начальные данные (4.1) быстро стабилизируются на бесконечности при $s^{ \pm} \rightarrow \pm \infty$ согласно (2.6). Поэтому решения также стабилизируются, причем к той же самой константе, так как в уравнении (4.2) сохраняется интеграл

$$
\lim _{s \rightarrow \pm \infty} A_{ \pm}(s, \tau)= \pm \int_{-\infty}^{\infty} \partial_{s} A_{ \pm}(s, \tau) d s=-\ln a^{2} .
$$

Структура быстро убывающих остатков сохраняется в решениях уравнений первого порядка $A_{ \pm}(s, \tau)=-\ln a^{2}+O\left(s^{-N}\right), s \rightarrow \pm \infty$, поскольку они сводятся к обькновенным дифференциальным уравнениям вдоль характеристик. Это свойство обеспечивает выполнение условия (2.6) при $\tau>0$. Следовательно, полученная пара $A_{ \pm}\left(s^{ \pm}, \tau\right)$ при всех значениях параметра $\tau \in\left[0, \tau_{0}\right]$ определяет решение уравнения Лиувилля, которое берется в качестве главного члена $\stackrel{0}{u}$.

Вычисленная по $\stackrel{0}{u}$ правая часть $\stackrel{1}{f}$ убывает при $|x|+t \rightarrow \infty$ равномерно по $\tau$ в силу условий (4.2). Тогда согласно лемме 3.3 линеаризованная задача для поправки имеет ограниченное решение.

СлЕДСТВИЕ 4.1. Построенное таким способом $Ф А Р U_{1}=\stackrel{0}{u}+\varepsilon \stackrel{1}{u}$ дает в уравнениях (0.2), (0.3) невязку порядка $O\left(\varepsilon^{2}\right)$ равномерно по $0<t \leqslant \tau_{0} \varepsilon^{-1}$.

Объединяя доказанные утверждения, получаем 
ТЕОремА 4.1. Пусть оператор возмущения имеет вид (0.4), а начальные данные в (0.3) таковы, что уравнения (2.1) не находятся на спектре. Тогда в задаче о возмущении уравнения Лиувилля (0.2), (0.3) главный член ФАР определяется формулой (0.1) в которой параметры $r_{ \pm}\left(s^{ \pm}, \varepsilon t\right)$ зависят от двух переменных. Зависимость их от медленного времени находится из уравнений (4.1)-(4.3) посредством форм⿻л (2.8)-(2.11).

5. Построение поправок в $\Phi$ АР. Метод двухмасштабных разложений в задачах для обькновенных дифференциальных уравнений зачастую позволяет получить полное асимптотическое разложение решения в виде бесконечных рядов. Перенос тех же идей на уравнения в частных производных обеспечивает получение главного члена и иногда первых поправок. В старших поправках метод двух масштабов может оказаться непригодным. Проанализируем этот подход для нашей задачи $(0.2),(0.3)$.

Рекуррентная система уравнений (1.4) определяет поправки $\stackrel{n}{u}$ через главньй член. Если последний параметризован парой функций $A_{ \pm}$в форме $(2.11)$, то эти параметры естественно использовать и для поправок. Такую параметризацию можно записать в операторной форме

$$
\stackrel{n}{u}=\stackrel{n}{U}\left[A_{+}, A_{-}\right]
$$

если указать способ выделения решения линеаризованного уравнения (1.4), т. е. определить операторы $\stackrel{n}{\boldsymbol{U}}$. Мы будем использовать для поправок формулу (3.6). Таким образом, приходим к формальному решению

$$
u(x, t ; \varepsilon) \approx \Phi\left[A_{+}, A_{-}\right]+\sum_{n=1}^{\infty} \varepsilon^{n} \stackrel{n}{U}\left[A_{+}, A_{-}\right], \quad \varepsilon \rightarrow 0
$$

Важно отметить, что конструирование ФАР в виде отрезка этого ряда возможно до тех пор, пока решение Лиувилля, взятое в качестве главного члена, выражается через $A_{ \pm}$, т. е. при выполнении условий согласования в асимптотиках (2.6).

Для построение $\Phi$ АР порядка $N$, пригодного вплоть до далеких времен $t \approx \varepsilon^{-1}$, требуется исключить секулярности в соответствующих поправках. Для этого на каждом шаге следует предусмотреть произволы в виде неопределенной пока зависимости от медленного времени $\tau$. Разные варианты двухмасштабных методов дают разные рецепты решения этой проблемы. Наиболее общим является подход Крылова-Боголюбова, основу которого составляет асимптотическое разложение для параметров главного члена

$$
A_{ \pm}\left(s^{ \pm}, \tau ; \varepsilon\right) \approx \sum_{n=0}^{\infty} \varepsilon^{n} \stackrel{n}{A}_{ \pm}\left(s^{ \pm}, \tau\right), \quad \varepsilon \rightarrow 0 .
$$

В таком подходе дело сводится к нахождению коэффициентов этого разложения $\stackrel{n}{A}_{ \pm}\left(s^{ \pm}, \tau\right)$ как функций двух переменных. Остается выписать уравнения и начальные условия для них.

Чтобы исключить секулярности сразу из всех поправок, достаточно потребовать

$$
\pm 2 \partial_{\tau} \partial_{s} A_{ \pm}-\boldsymbol{F}_{ \pm}\left[A_{ \pm}\right]+\varepsilon \partial_{\tau}^{2} A_{ \pm}=0
$$


Операторы $F_{ \pm}$здесь определяются через возмущение

$$
\boldsymbol{F}_{ \pm}\left[A\left(s^{ \pm}, \tau\right)\right]=\boldsymbol{F}[-4 t+A(x \pm t, \varepsilon t)]
$$

с учетом замены производных: $\partial_{t} \Longrightarrow \pm \partial_{s}+\varepsilon \partial_{\tau}, \partial_{x} \Longrightarrow \partial_{s}$. Нетрудно проверить, что требования (5.4) обеспечивают разрешимость рекуррентной системы линеаризованных уравнений (1.4) в классе убывающих функций. Получаемые приближения записьваются в параметрической форме (5.1) через известные операторы $\stackrel{n}{\boldsymbol{U}}$. Сами параметры $A_{ \pm}$ остаются пока неопределенньми.

Убывающие на бесконечности поправки $\stackrel{n}{u}$ дают невязки в начальные уравнения (0.3). Эти невязки будем компенсировать за счет выбора начальных значений для главного члена $\Phi$ АР. Попросту говоря, выполнение начальных условий (0.3) требуется от полного $\Phi$ АР (5.2), так что начальные уравнения (0.3) переходят в пару начальных условий для функций $A_{ \pm}(x \pm t, \tau ; \varepsilon)$ :

$$
\begin{array}{r}
\Phi\left[A_{+}, A_{-}\right]+\sum_{n=1}^{\infty} \varepsilon^{n} \stackrel{n}{\boldsymbol{U}}\left[A_{+}, A_{-}\right] \approx \psi_{0}(x) \quad \text { при } t=\tau=0, \\
{\left[\partial_{t}+\varepsilon \partial_{\tau}\right]\left(\Phi\left[A_{+}, A_{-}\right]+\sum_{n=1}^{\infty} \varepsilon^{n} \stackrel{n}{\boldsymbol{U}}\left[A_{+}, A_{-}\right]\right) \approx \psi_{1}(x) \quad \text { при } t=\tau=0 .}
\end{array}
$$

Описанньй подход напоминает известный метод погранслоя в интерпретации [18, c. 31] с той разницей, что внешнее разложение (5.3) связьвается с погранслоем посредством нелинейных операторов в (5.5), (5.6).

Таким образом, исходная задача (0.2), (0.3) сведена к системе (5.4) с условиями (5.5), (5.6). В постановке задачи для функций $A_{ \pm}$используются также условия на бесконечности, которые получаются из соображений согласования с асимптотикой невозмущенного решения (2.5):

$$
A_{ \pm}\left(s^{ \pm}, \tau ; \varepsilon\right) \rightarrow 0, \quad s^{ \pm} \rightarrow \mp \infty .
$$

Полученные уравнения (5.4)-(5.7) описывают медленную деформацию параметров $A_{ \pm}\left(s^{ \pm}, \tau ; \varepsilon\right)$. Они содержат малый параметр $\varepsilon$ и могут решаться в асимптотическом приближении. Проблема лишь в выполнении дополнительных условий (2.6), необходимых для представления решения Лиувилля через $A_{ \pm}$. Оказывается, что эти условия можно гарантировать на первых шагах при дополнительных ограничениях на возмущение.

Tеорема 5.1. Пусть оператор возмущения имеет вид (0.4) с $F_{2} \equiv 0$, и начальные функиии в (0.3) таковы, что уравнения (2.1) не находятся на спектре. Тогда в задаче о возмущении уравнения Лиувилля (0.2), (0.3) метод двухмасштабных разлохсений дает ФАР порядка $N=2$ в виде отрезков рядов (1.1), (1.3).

ДокАЗАТЕльство. Если для задачи (5.5)-(5.7) строить ФАР в форме (5.3), то для коэффициентов $\stackrel{n}{A}_{ \pm}\left(s^{ \pm}, \tau\right)$ получается рекуррентная система дифференциальных уравнений с начальными условиями при $\tau=0$ и краевьпи условиями на бесконечности при $s^{ \pm} \rightarrow \mp \infty$. 
На исходном шаге $n=0$ приходим к рассмотренной ранее задаче (4.1)- (4.3). Разрешимость ее на некотором достаточно малом промежутке $\tau \in\left[0, \tau_{0}\right]$ установлена в лемме 4.1.

Для следующих приближений $\stackrel{n}{A} \pm(s, \tau)$ получаются линеаризованные уравнения

$$
\pm 2 \partial_{\tau} \stackrel{n}{A}_{ \pm}-\delta H_{ \pm}\left(\partial_{s} \stackrel{0}{A}_{ \pm}\right) \partial_{s} \stackrel{n}{A}_{ \pm}=\stackrel{n}{g}{ }_{ \pm}(s, \tau)
$$

Правые части вычисляются через предыдущие приближения; например, на первом шаге

$$
\stackrel{1}{g} \pm=\partial_{w} F_{1}(v, w) \partial_{\tau} \stackrel{0}{A_{ \pm}}-\int_{\mp \infty}^{s} \partial_{\tau}^{2} \stackrel{0}{A}_{ \pm}(\eta, \tau) d \eta
$$

при $v=\partial_{s} \stackrel{0}{A_{ \pm}}, w=-4 \pm \partial_{s} \stackrel{0}{A_{ \pm}}$.

Уравнения дополняются начальными данными

$$
\stackrel{n}{A}_{ \pm}(s, 0)=\stackrel{n}{A}_{ \pm, 0}(s), \quad s \in \mathbb{R}
$$

которые также определяются рекуррентными формулами. Например, на первом шаге функции $\stackrel{1}{A}{ }_{ \pm, 0}$ вычисляются через значения первой поправки $\stackrel{1}{u}(x, t, \tau)$ и ее производные при $t=\tau=0$. Линейные задачи (5.8), (5.9) разрешимы на том же промежутке $\tau \in\left[0, \tau_{0}\right]$.

На первом шаге правые части в дифференциальных уравнениях (5.9) имеют согласованную асимптотику $\stackrel{1}{g}_{ \pm}(s, \tau)=O\left(s^{-N}\right)$ при $s^{ \pm} \rightarrow \pm \infty$, поскольку в силу уравнения для главного члена (4.2) и с учетом стабилизации на бесконечности функций $\partial_{s} \stackrel{0}{A} \pm$ $O\left(s^{-N}\right)$ обращается в нуль интеграл

$$
\int_{-\infty}^{\infty} \partial_{\tau}^{2} \stackrel{0}{A_{ \pm}}(s, \tau) d s=\int_{-\infty}^{\infty}\left[H_{ \pm}^{\prime}\left(\partial_{s} \stackrel{0}{A} A_{ \pm}\right)\right]^{2} \partial_{s}^{2} \stackrel{0}{A}_{ \pm}(s, \tau) d s=0
$$

Поэтому решения уравнений (5.9) с согласованными начальными данными будут иметь согласованную асимптотику при $s^{ \pm} \rightarrow \pm \infty$, так что для $\stackrel{1}{A} \pm$ выполняется свойство (2.6). Тем самьм оправдана конструкция $\Phi$ АР (5.2) на первых двух шагах. Функция

$$
U_{2}(x, t ; \varepsilon)=\Phi\left[A_{+}, A_{-}\right]+\varepsilon \stackrel{1}{U}\left[A_{+}, A_{-}\right]+\varepsilon^{2} \stackrel{2}{U}\left[A_{+}, A_{-}\right]
$$

определена при $A_{ \pm}=\stackrel{0}{A_{ \pm}}\left(s^{ \pm}, \tau\right)+\varepsilon \stackrel{1}{A}_{ \pm}\left(s^{ \pm}, \tau\right), \tau=\varepsilon t, s^{ \pm}=x \pm t$, и дает невязки в уравнениях $(0.2),(0.3)$ порядка $O\left(\varepsilon^{3}\right)$ равномерно при $0<t \leqslant \tau_{0} \varepsilon^{-1}$.

Переход к $\Phi$ АР в форме (1.1)-(1.3) не представляет проблемы ввиду тривиальности уравнений $(2.9),(2.10)$, связьваюших $A_{ \pm}$с парой $r_{ \pm}$. Теорема доказана.

На следующих шагах свойство согласования в асимптотиках (2.6) может нарушаться; метод двух масштабов становится неэффективньм. 
6. Заключение. Использованный выше метод двухмасштабных разложений весьма популярен и иногда позволяет получить полное асимптотическое разложение решения в виде бесконечных рядов. Однако, для уравнений в частных производных, как правило, возникают непреодолимые трудности при нахождении старших поправок, см. например, [11] (если только не строить специальные ФАР [8]-[10]). Возникающие проблемы связаны с существом дела: в разных областях пространственно-временньх переменных решение имеет разную асимптотическую структуру и зависит от разных быстрых и медленных переменных. В таких ситуациях подходящей альтернативой является метод согласования асимптотических разложений [18]. Однако, он довольно громоздок и при вычислении главного члена $\Phi$ АР не дает преимуществ.

Поясним это на рассмотренной задаче. Ввиду леммы 2.1 вся информация о главном члене $\Phi$ АР на далеких временах $t \gg 1$ содержится в паре функций $A_{ \pm}\left(s^{ \pm}, \varepsilon t\right)$. Поэтому при $t \gg 1$ нет нужды использовать формулу $(0.1)$, и асимптотическое решение можно представить как сумму асимптотик по разньп областям за вычетом их общей части $-\ln a^{2}$ в виде: $u(x, t, \varepsilon) \approx-4 t+\ln a^{2}+A_{+}\left(s^{+}, \tau\right)+A_{-}\left(s^{-}, \tau\right), \varepsilon \rightarrow 0$, при $1 \ll t \leqslant O\left(\varepsilon^{-1}\right)$. Эта формула представляет собой главный член $\Phi$ АР для волнового уравнения (в которое переходит исходное уравнение $(0.2)$ при $t \gg 1)$ с учетом возмущения. Решение Лиувилля в форме (2.11) используется здесь при $\tau=0$ лишь постольку, поскольку для $A_{ \pm}\left(s^{ \pm}, \tau\right)$ требуются начальные условия. Однако, на временах не слишком больших $0<t \ll O\left(\varepsilon^{-1}\right)$ именно выражение (2.11) дает главный член $\Phi$ АР. При этом параметры $A_{ \pm}$можно взять фиксированными (при $\left.\tau=0\right)$, т. е. использовать решение невозмущенного уравнения. Такое представление асимптотического решения в разной форме для разных областей соответствует методу согласования; в рассмотренной ситуации оно вьглядит излишним.

Преимущество этого подхода обнаруживается либо на следующих шагах при вычислении поправок, либо в случае недивергентных возмущений. В общей ситуации из-за деформации параметров может нарушаться условие согласования асимптотик (2.6) при $\tau>0$. В таком случае главньй член $\Phi$ АР не представляется через решение Лиувилля. Но на далеких временах такое представление не нужно, поскольку сами функции $A_{ \pm}\left(s^{ \pm}, \tau\right)$ определяют главньй член $\Phi$ АР. Согласование с решением Лиувилля в области не слишком больших времен получается в силу выбора начальных данных $A_{ \pm}\left(s^{ \pm}, 0\right)$.

Следует указать, что в общей ситуации возмущение влияет не только на медленную деформацию быстрых волн $A_{ \pm}(s, \tau)$. Рассогласование асимптотик при $s^{ \pm} \rightarrow \pm \infty$ свидетельствует о влиянии возмущения в секторе "хвостов" между расходящимися волнами, где $s^{+} \gg 1, s^{-} \ll-1$. В этой области $\Phi$ АР представляется функцией двух медленных переменных $\xi=\varepsilon x, \tau=\varepsilon t$. Похожая проблема для случая слабо нелинейных гиперболических систем была исследована автором в [19]. Детальная реализация метода согласования для задачи $(0.2),(0.3)$ выходит за рамки данной статьи.

\section{СПИСОК ЦИТИРОВАННОЙ ЛИТЕРАТУРЫ}

[1] Калякин Л.А.Длинноволновые асимптотики. Интегрируемые уравнения как асимптотический предел нелинейных систем // УМН. 1989. Т. 44. № 1. С. 3-34.

[2] Карпман В.И., Маслов Е. М. Теория возмущений для солитонов // ЖЭТФ. 1977. T. 73. № 8. C. 538-559.

[3] Keener J. P., Mc-Loughlin D.W. Solitons under perturbations // Phys. Rev. A. 1978. V. 18. № 4. P. 1652-1680. 
[4] Маслов Е. М. К теории возмущений для солитонов во втором приближении // Теорет. и матем. физика. 1980. Т. 42. №3. С. 362-370.

[5] Кричевер И. М. Гессианы интегралов уравнения Кортевега-де Фриза и возмущение конечнозонных решений // Докл. АН СССР. 1983. Т. 270. №6. С. 1312-1316.

[6] Ньюэлл А. Обратное преобразование рассеяния // Солитоны / ред. Р. Буллафф, Ф. Кодри. М.: Мир, 1983. С. 193-269.

[7] Dobrohotov S. Yu., Maslov V. P. Multiphase asymptotics of nonlinear partial differential equations with a small parameter // Sov. Sci. Rev. V. 3, 1988. P. 221-311.

[8] Маслов В. П., Омельянов Г. А. Об условиях типа Гюгонио для бесконечно узких решений уравнения простых волн // Сиб. матем. ж. 1983. Т. 24. С. 172-182.

[9] Маслов В.П., Омельянов Г.А. Солитонообразные асимптотики внутренних волн в стратифицированной жидкости с малой дисперсией // Дифференц. уравнения. 1985. Т. 21. № 10. С. $1766-1775$.

[10] Маслов В. П. Асимптотические методы решения псевдодифференциальных уравнений. М.: Наука, 1987.

[11] Калякин Л.А. Возмущение солитона Кортевега-де Фриза // Теорет. и матем. физика. 1992. Т. 92. № 1. С. $62-76$.

[12] Погребков А.К., Поливанов М.К. Теория поля Лиувилля // Тр. МИАН. 1987. T. 176 . C. $86-96$.

[13] Джорджадзе Г. П., Погребков А.К., Поливанов М.К. Сингулярные решения уравнения $\square \varphi+\left(m^{2} / 2\right) \exp \varphi=0$ и динамика особенностей // Теоретич. и матем. физика. 1979. T. 40. № 2. C. 221-234.

[14] Liouville J. Sur l'équation aux différences partielles $\partial^{2} \ln \lambda / \partial u \partial v \pm \lambda^{2} q=0 / /$ J. Math. Pure et Appl. 1853. V. 18. P. 71-74.

[15] Боголюбов Н. Н., Митропольский Ю. А. Асимптотические методы в теории нелинейных колебаний. М.: Наука, 1974.

[16] Bianchi L. // Ann. Sci. Norm. Super. Piza Ser. 1. 1879. V. 26. № 2.

[17] Погребков А. К. О глобальных решениях задачи Коши для уравнения Лиувилля $\varphi_{t t}-\varphi_{x x}$ $=-1 / 2 m^{2} \exp \varphi$ в случае сингулярных начальных данных // Докл. АН СССР. 1979. Т. 244. № 4. C. $873-876$.

[18] Ильин А. М. Метод согласования асимптотических разложений решений краевых задач. M.: Наука, 1989.

[19] Калякин Л. А. Длинноволновая асимптотика решения гиперболической системы уравнений // Матем. сб. 1984. Т. 124. №1. С. 96-120. 